\title{
Role of lordotic rod contouring in thoracic myelopathy: a technical note
}

\author{
Kalyan Kumar Varma Kalidindi ${ }^{1} \cdot$ Harvinder Singh Chhabra ${ }^{1} \cdot$ Mohd Rafiq Bhat $^{1} \cdot$ Sulaiman Sath $^{1}$ • \\ Abhishek Mannem ${ }^{1}$
}

Received: 13 May 2019 / Revised: 29 July 2019 / Accepted: 3 August 2019

(c) International Spinal Cord Society 2019

\begin{abstract}
Introduction Decompressive surgery for thoracic myelopathy due to anterior pathology can be challenging. Direct decompression through anterior approaches is associated with approach-related complications, whereas anterior decompression through posterior approaches is technically demanding and may result in neurological deterioration. We present a simple and effective surgical technique of indirect decompression through lordotic rod contouring to reduce such complications.

Case presentation Patients who presented to our center between March 2016 and March 2017 with symptoms and signs suggestive of thoracic myelopathy predominantly due to anterior pathologies such as ossification of the thoracic posterior longitudinal ligament, posterior bony spur, and thoracic disc herniation were evaluated in our study. The indications for surgical treatment were progressive neurological impairment and severe myelopathy (grade III or more on Nurick grade). Only those patients classified as grade III and above on American Society of Anaesthesiologists (ASA) physical status scale were included in the study. All the cases were operated by a single surgeon by a posterior-only approach. We have used this technique in four patients with thoracic myelopathy due to combined or predominant anterior pathology. Postoperative imaging confirmed adequate decompression of the spinal cord. All the cases improved substantially in terms of clinical outcome.

Discussion This surgical technique could be a useful alternative to direct anterior decompression in patients who present with symptoms of progressive severe myelopathy due to anterior compression and could be the standard of care in those at high risk for major surgery.
\end{abstract}

\section{Introduction}

Thoracic myelopathy is less common disorder as compared with cervical myelopathy [1]. Degenerative spine disorders, including intervertebral disc herniations (IDH), ossification of the posterior longitudinal ligament(OPLL), posterior bone spurs, and ossification of ligamentum flavum (OLF) are among the major causes of thoracic myelopathy [2]. The natural kyphosis of the thoracic spine causes the spinal cord to lie directly on the posterior vertebral bodies and the posterior longitudinal ligaments in this region. Also, the

Kalyan Kumar Varma Kalidindi

kalyanvarmambbs@gmail.com

1 Indian Spinal Injuries Center, Vasant Kunj, New Delhi 110070 , India denticulate ligaments running longitudinally between the spinal cord and nerve roots limit posterior displacement of the spinal cord within the canal and make the spinal cord more sensitive to anterior compression [3].

Surgery is often indicated for thoracic myelopathy. The approach is usually dictated by the location and type of pathology [4]. Posteriorly based pathology e.g., OLF and facet hypertrophy are better dealt by laminectomy and if required, a medial facetectomy. If the stenosis is lateral or anterolateral, a laminectomy alone is inadequate and more aggressive approaches like pediculectomy, costotransversectomy, lateral extracavitary approach, or even ventral decompression may be necessary. They may increase the morbidity of the procedure and also the risk of neurological deterioration [5]. Often, the general condition of the patients would not allow such major procedures to be undertaken [5]. The authors present a simple and effective technique of indirect decompression using a rod contouring technique 


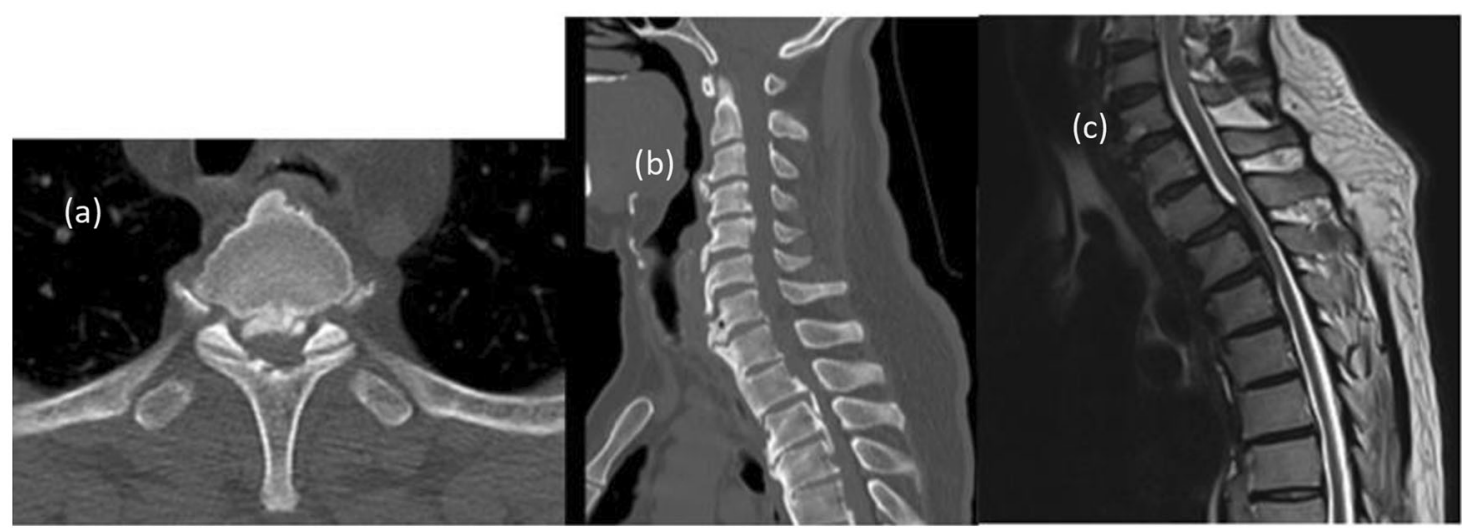

Fig. 1 Axial cut at T3 level on CT scan (a), midsagittal cut on CT scan (b), as well as T2 weighted sagittal MRI scan (c) images of the thoracic spine of a 70-year-old male patient (case 1) showing ossification of the thoracic posterior longitudinal ligament behind T2 to T4 levels with anterior compression of the cord and associated signal intensity changes at the same level along with laminectomy for addressing anterior pathology like OPLL or IDH in thoracic stenosis. This technique could be particularly useful when the patient cannot tolerate a major procedure.

\section{Case presentation}

Patients who presented to our center between March 2016 and March 2017 with symptoms and signs suggestive of thoracic myelopathy predominantly due to anterior pathologies such as ossification of the thoracic posterior longitudinal ligament, posterior bony spur, and thoracic disc herniation were included in our study. The indications for surgical treatment were progressive neurological impairment and severe myelopathy (grade III or more on Nurick grade). Patients classified as grade III and above on American Society of Anaesthesiologists physical status scale were considered candidates for this procedure. The patients underwent a thorough preoperative workup and written informed consent was taken detailing the possibility of a second procedure at a later date, in case the recovery would not be as expected. All the cases were operated by a single surgeon by a posterior-only approach.

\section{Illustrative cases}

\section{Case 1}

\section{History}

A 70-year-old man was referred to our hospital with a 6 month history of gait imbalance. The symptoms had rapidly progressed in the last 3 months. There was no history of trauma and there were no constitutional symptoms. His medical history included chronic renal failure requiring dialysis, chronic obstructive pulmonary disease, diabetes, and hypertension.

\section{Examination}

On examination, the patient was wheelchair bound (Nurick grade V). The muscle strength was decreased in both lower limbs [lower extremity motor score of 23 out of 50 as per International Standards for Neurological Classification of Spinal Cord Injury (ISNCSCI)]. There was decreased sensation below T5 (sensory grading 1 to pinprick as well as light touch as per ISNCSCI). The neurological level was T5. The reflexes were exaggerated in both lower limbs (grade $3+$ knee and ankle reflexes) and there was an extensor plantar response. MRI of the thoracic spine with whole spine screening and CT scan of the thoracic spine suggested thoracic stenosis with OPLL (Fig. 1). Decompressive surgery by a posterior approach was planned.

\section{Surgical details}

The patient was positioned prone with appropriate padding of all bony prominences and ensuring that the spine was aligned with the hips extended. A midline approach was used with subperiosteal dissection of the affected region. After confirming levels with intraoperative imaging, the spinous processes and interspinous ligament were removed with a rongeur. Pedicle screw entry points were identified and a pilot hole was made using a $3 \mathrm{~mm}$ burr. A Lenke probe used to create a track and accuracy was checked with a sound. Subsequently, $5.5 \mathrm{~mm}$ size pedicle screws were placed from $\mathrm{T} 1$ to $\mathrm{T} 5$ under fluoroscopic guidance. A highspeed burr was used to create bilateral troughs at the lamina- 


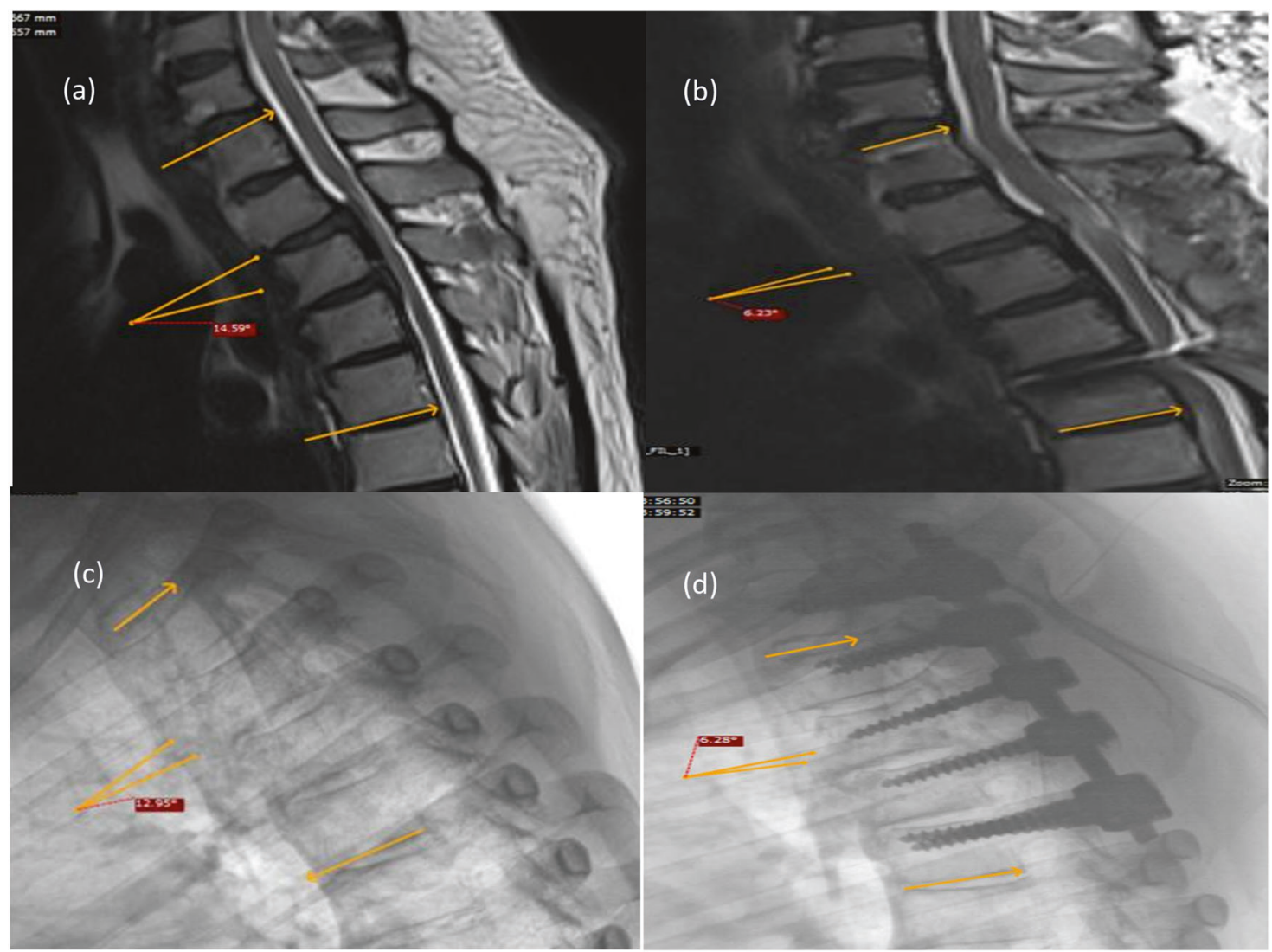

Fig. 2 Preoperative (a) and postoperative (b) T2 weighted MRI images of the patient (case 1) showing adequate fall back of the cord. CSF column could be appreciated in front of the involved spinal cord. The segmental kyphosis at the level of instrumentation (T1 to T5) decreased from $14.6^{0}$ preoperatively to $6.2^{0}$ postoperatively. Preoperative (c) and

facet junction and remove the laminae en bloc. Kerrison Rongeurs were used to widen the laminectomy and a partial facet resection was performed. A microsurgical technique was used to separate the ligamentum flavum from the dura. The rods were prebent based on the preoperative calculation of segmental kyphosis on imaging to achieve a lordotic effect at the instrumented level. A rod pusher and a rocker blocker were used to sequentially push the rod into each screw. The appropriate placement was checked with fluoroscopy. Posterolateral fusion was performed using bone chips of the resected laminae and spinous processes. The neurological status of the patient was monitored by motor evoked potentials after each screw placement, during laminectomy, as well as ligamentum flavum removal and during rod placement. Finally, the wound was closed over a negative suction drain after adequate hemostasis.

\section{Postoperative course}

The immediate postoperative period assessment revealed no deterioration in neurological status. Postoperative X-rays postoperative (d) X-ray images of the patient (case 1) showing that the segmental kyphosis between T2 to T5 decreased from $13^{0}$ preoperatively to $6.3^{0}$ postoperatively (we chose T2 level as the superior endplate of T1 vertebra could not be appreciated well on lateral pre and postoperative $\mathrm{X}$-ray images)

showed appropriately placed screws and adequate fixation of the implant with relative segmental lordosis at the index level (Fig. 2). Postoperative MRI showed adequate relative lordosis of the involved segment and CSF column anteriorly suggesting adequate decompression (Fig. 2).On follow up assessment at 6 months postoperatively, he had an improved Nurick score and was able to walk with slight difficulty (Nurick II). The patient was doing well at 2-year follow up.

Case 2

A 68-year-old woman presented to our center with complaints of progressive difficulty in walking for 3 months. Past medical history was significant for diabetes, hypertension, rheumatoid arthritis with interstitial lung disease, and postpolio residual paralysis of the left lower limb. There was recent onset weakness (lower extremity motor score of 32 out of 50 as per ISNCSCI) and hypoalgesia in the lower extremities (sensory grade 1 to pinprick and light touch as per ISNCSCI from T10 downwards). The neurological level was T9. The symptoms and signs suggested thoracic myelopathy with Nurick grade III.CT scan 


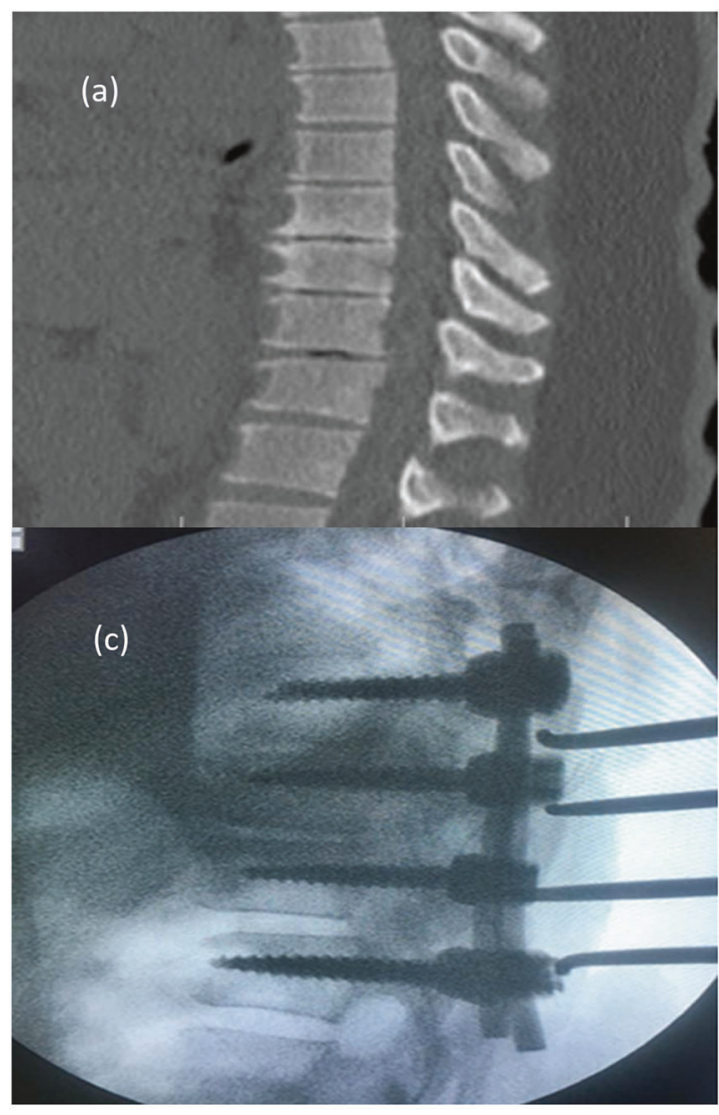

Fig. 3 a Midsagittal cut on CT scan of the thoracic spine of a 68-yearold woman (case 2) showing degenerative changes in the thoracic spine, kyphotic alignment of the thoracic spine, and mobile intervertebral disc spaces in the thoracic spine. b Sagittal T2 weighted MRI of the thoracic spine of a 68-year-old woman (case 2) showing multilevel intervertebral disc prolapse (T8-T9/T9-T10 and T10-T11 levels)

and MRI scan revealed a multilevel intervertebral disc prolapse at T8-T9/T9-T10 and T10-T11 level with signal intensity changes at T9-T10 and T10-T11 levels (Fig. 3). The surgery was performed by the same procedure as described above with fixation from T8 to T11 levels (Fig. 3). The postoperative X-ray and MRI images showed adequate relative lordosis and decompression of the thoracic spinal cord (Fig. 4). Both muscle strength and sensation of the lower extremities returned to near normal. She was able to walk without support at 3 months follow up (Nurick grade I). She was doing well at 2 years follow up.

Cases 3 and 4 are described in Figs. 5, 6.

\section{Results}

We used this technique in four patients who had thoracic myelopathy due to predominant anterior pathology in the thoracic spine. No major intraoperative or postoperative surgical complications were encountered in any patient. All

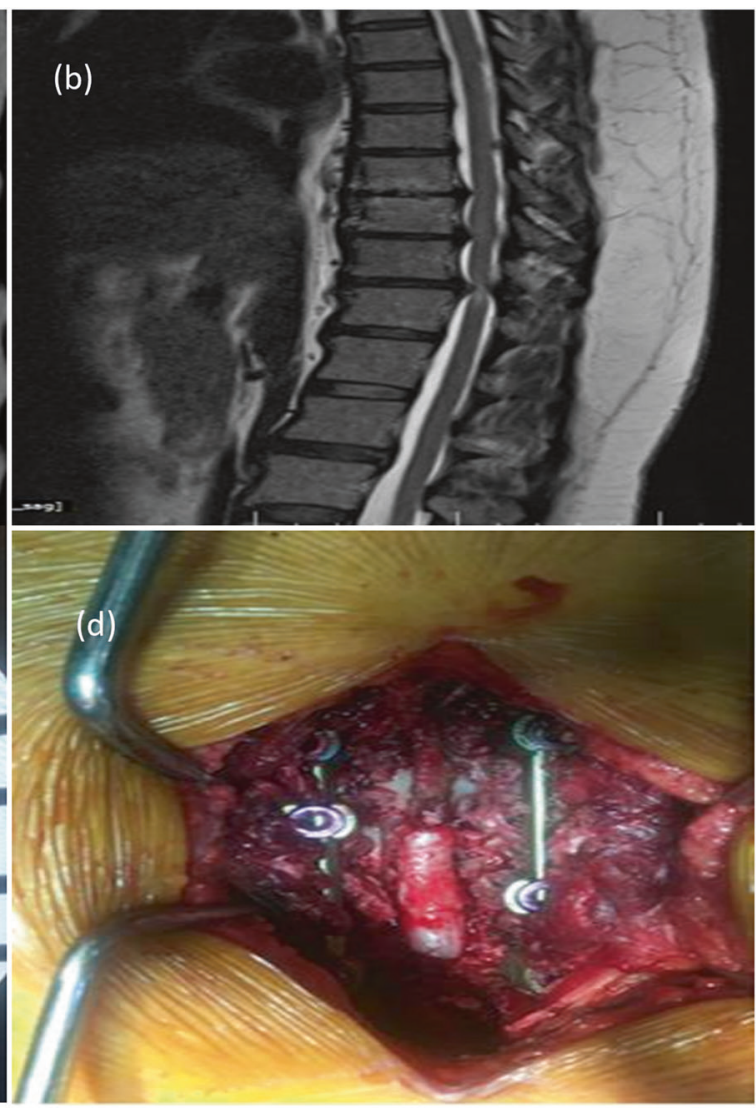

with signal intensity changes at T9-T10 and T10-T11 levels suggesting thoracic myelopathy. c Intraoperative $\mathrm{C}$-arm image of the thoracic spine of the patient (case 2) showing lordotic rod fixation. d Intraoperative clinical image of the patient (case 2) showing decompressed thoracic cord using the microsurgical technique and instrumentation at the same level

the patients had an improvement in Nurick grade on followup assessments and none of them required a second procedure.

\section{Discussion}

Conservative management is usually ineffective for thoracic myelopathy. Hence, surgical treatment is necessary. The physiological kyphotic alignment of the thoracic spine places the spinal cord at more risk of anterior compression and posterior decompression by laminectomy alone and may not adequately relieve the anterior pressure on the spinal cord [6]. However, the relative avascularity and the less space available to the thoracic spinal cord results in poor results of attempted direct decompression by surgical removal of the anterior pathology. Anterior decompression via an anterior approach is technically challenging and patients with preexisting severe systemic cardiopulmonary diseases 


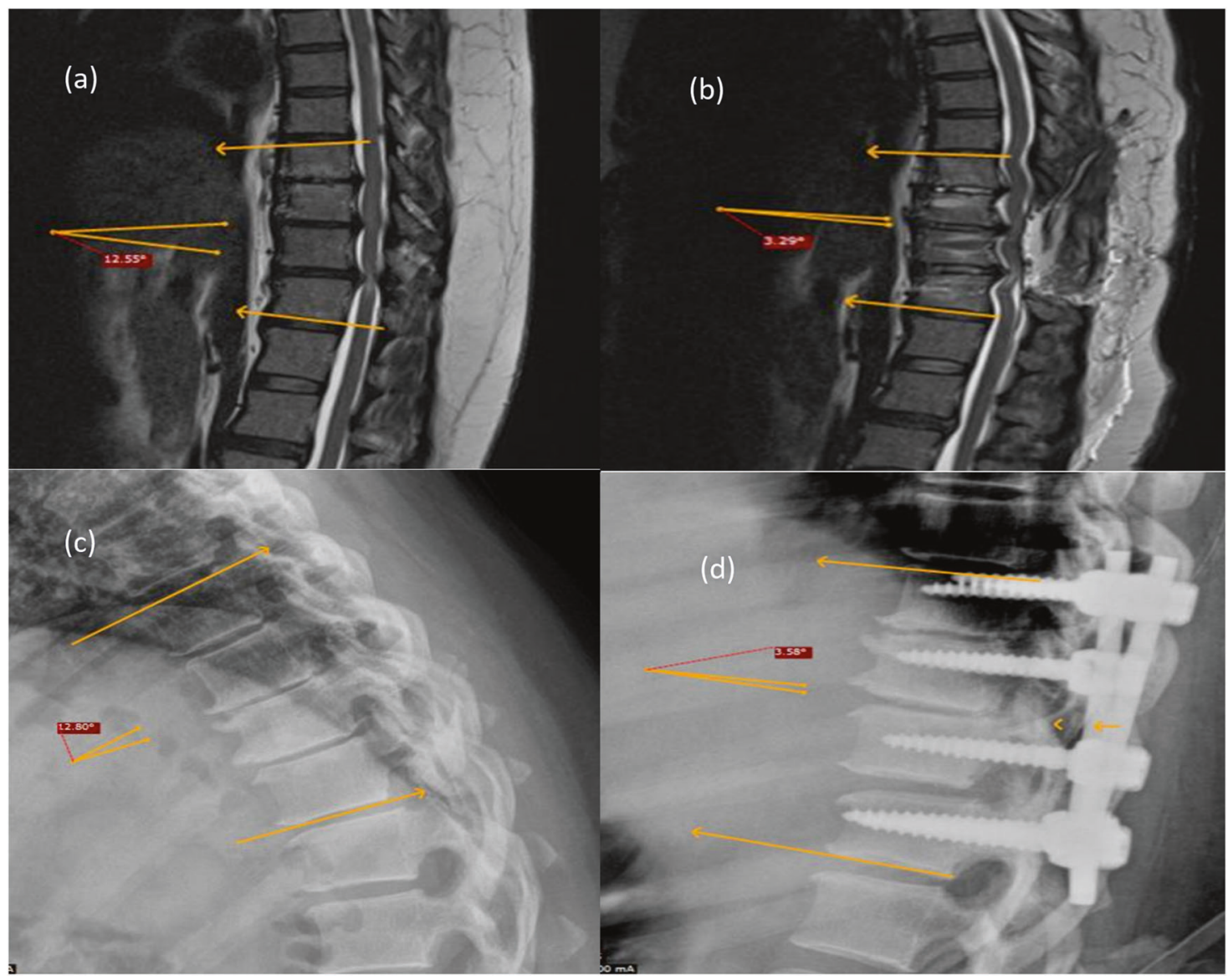

Fig. 4 Preoperative (a) and postoperative (b) T2 weighted MRI images of the patient (case 2) showing the decompressed spinal cord. The segmental kyphosis at the level of instrumentation (T8 to T11) decreased from $12.6^{0}$ preoperatively to $3.3^{0}$ postoperatively.
Preoperative (c) and postoperative (d) X-ray images of the patient (case 2) showing that the segmental kyphosis between T8 to T11 decreased from $12.8^{0}$ preoperatively to $3.6^{0}$ postoperatively may not tolerate the procedures well [7]. Otsuka et al. reported anterior decompression through a posterior approach but it carries a high risk of neurological deterioration (33\% of their cases) [8]. Tomita et al. described a circumspinal decompression technique using a staged anterior and posterior approach [9]. Tsuzuki et al. described an extensive cervicothoracic laminoplasty decompression for OPLL but reported unsatisfactory outcome with residual impingement [10]. They proposed a staged spinal cord decompression through a posterior approach but the preliminary results did not guarantee a satisfactory outcome [6]. Yamazaki et al. reported transient paraparesis after laminectomy for thoracic myelopathy due to OPLL [11]. They reported a considerable degree of neurological recovery by posterior decompression and instrumented fusion despite persistent impingement of the anterior OPLL [12]. Matsuyama et al. reported good outcomes of surgery by expansive laminoplasty combined with instrumentation in OPLL, regardless of the type of ossification [13]. They proposed consideration of an additional anterior decompression after 6 months if the improvement is not satisfactory [14]. Kawahara et al. proposed a posterior decompression and fusion as the first step followed by an MRI at 3 weeks after surgery and additional decompressive fusion if decompression was insufficient on MRI [15].

Cord drift back is a described phenomenon after posterior surgery for cervical myelopathy. We assume that decompression along with relative lordosis achieved with instrumentation resulted in the cord drift back and clinical improvement in our cases. The amount of relative lordosis achieved was not more than ten degrees in any of our cases. The patients could still improve substantially in terms of clinical outcome. We also assume that the less preoperative kyphosis (none greater than 150) may also have contributed to our clinical results. The upper thoracic spine (or cervicothoracic junction) and lower thoracic spine (or thoracolumbar junction) with relatively less kyphosis may 


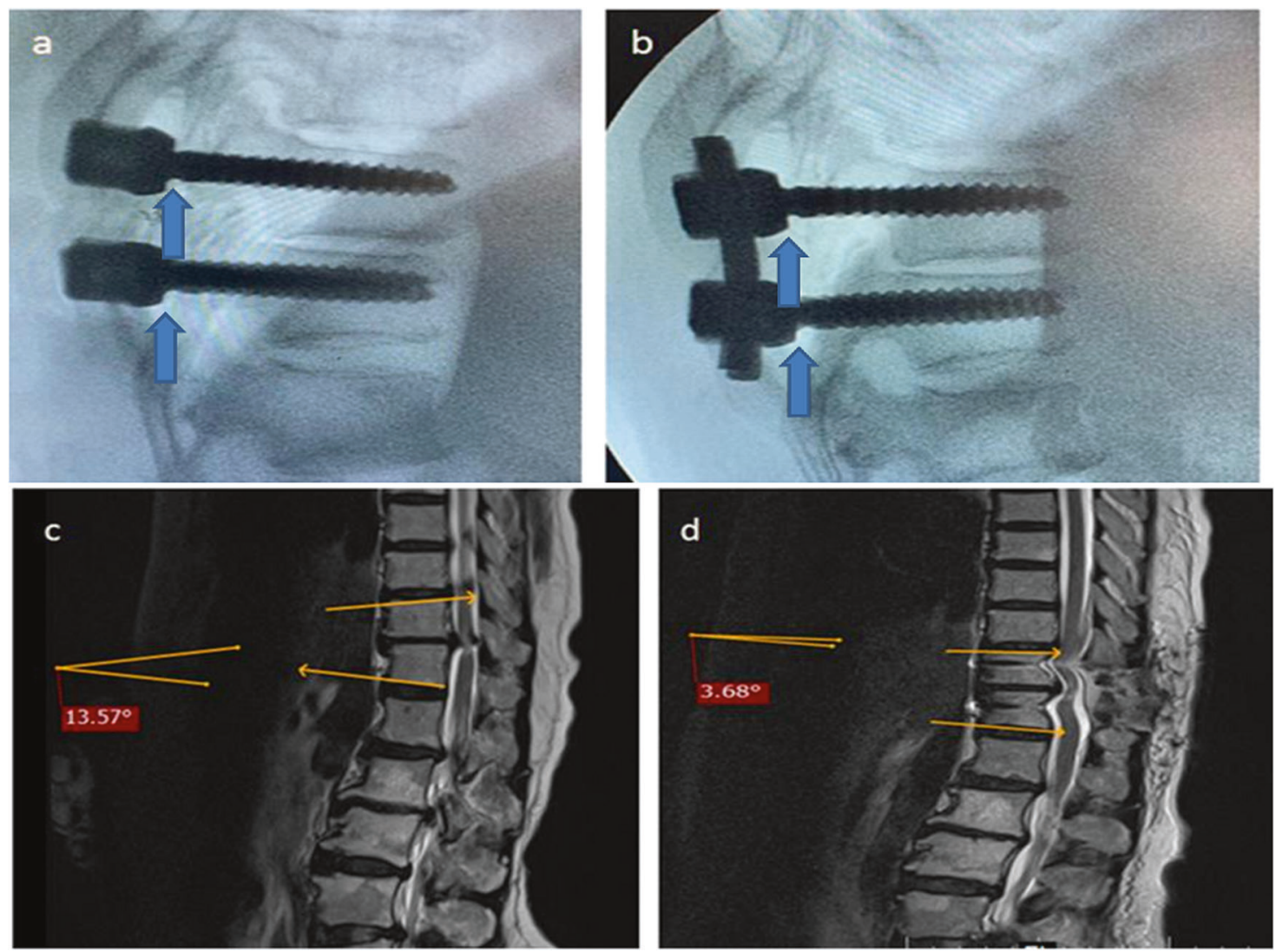

Fig. 5 Intraoperative C-arm images $(\mathbf{a}, \mathbf{b})$ of a 71-year-old female (case 3) with ASA-grade III with symptoms and signs of thoracic myelopathy showing the segmental relative lordosis achieved at the instrumented level after application of prebent rods. the relative position of the base of the screw heads (marked with blue arrows) can guide the intraoperative assessment of relative lordosis at the

particularly benefit with this technique. In our study, one patient had instrumentation in the upper thoracic spine and the other three at the lower thoracic spine.

The technique of indirect decompression by laminectomy and posterior instrumentation with lordotic rods has the advantages of less surgical stress, less approach related complications, less handling of cord with fewer chances of neurological deterioration, reduction of the dynamic component of myelopathy if any, and removal of associated posterior pathology, if present.

The main disadvantage of the technique is that there is no confirmatory evidence of adequate decompression during surgery. Intraoperative $\mathrm{C}$-arm images may guide us on the extent of relative lordosis achieved. The use of intraoperative ultrasound to assess the extent of decompression has been described but we do not have any experience with it. The second disadvantage is the chance of neurological deterioration, though less likely than other procedures. Though we did not encounter any case of instrumented level. Preoperative (c) and postoperative (d) sagittal MRI images show achievement of $10^{0}$ of relative lordosis at the instrumented level (T11-T12). Adequate decompression with continuous anterior CSF column could be appreciated in the postoperative images as compared preoperatively

neurological deterioration, neuromonitoring is recommended to avoid neurological complications during laminectomy and application of lordotic rods. Also, the use of temporary rods during decompression is advised to prevent neurological deterioration. The third disadvantage is the need for an additional anterior procedure if the recovery is not as expected. We found good improvement in all our cases on the last follow up and none of them required additional surgery for neurological recovery.

In conclusion, indirect decompression through lordotic rod contouring may be a useful alternative to direct anterior decompression in patients who present with symptoms of progressive severe myelopathy due to anterior cord compression. Ultimately, after further study, we believe it may be the standard of care in those at high risk for major surgery. Moreover, long term high quality studies with adequate sample size may establish the procedure as a standard of care in all patients with thoracic myelopathy due to anterior cord compression. 


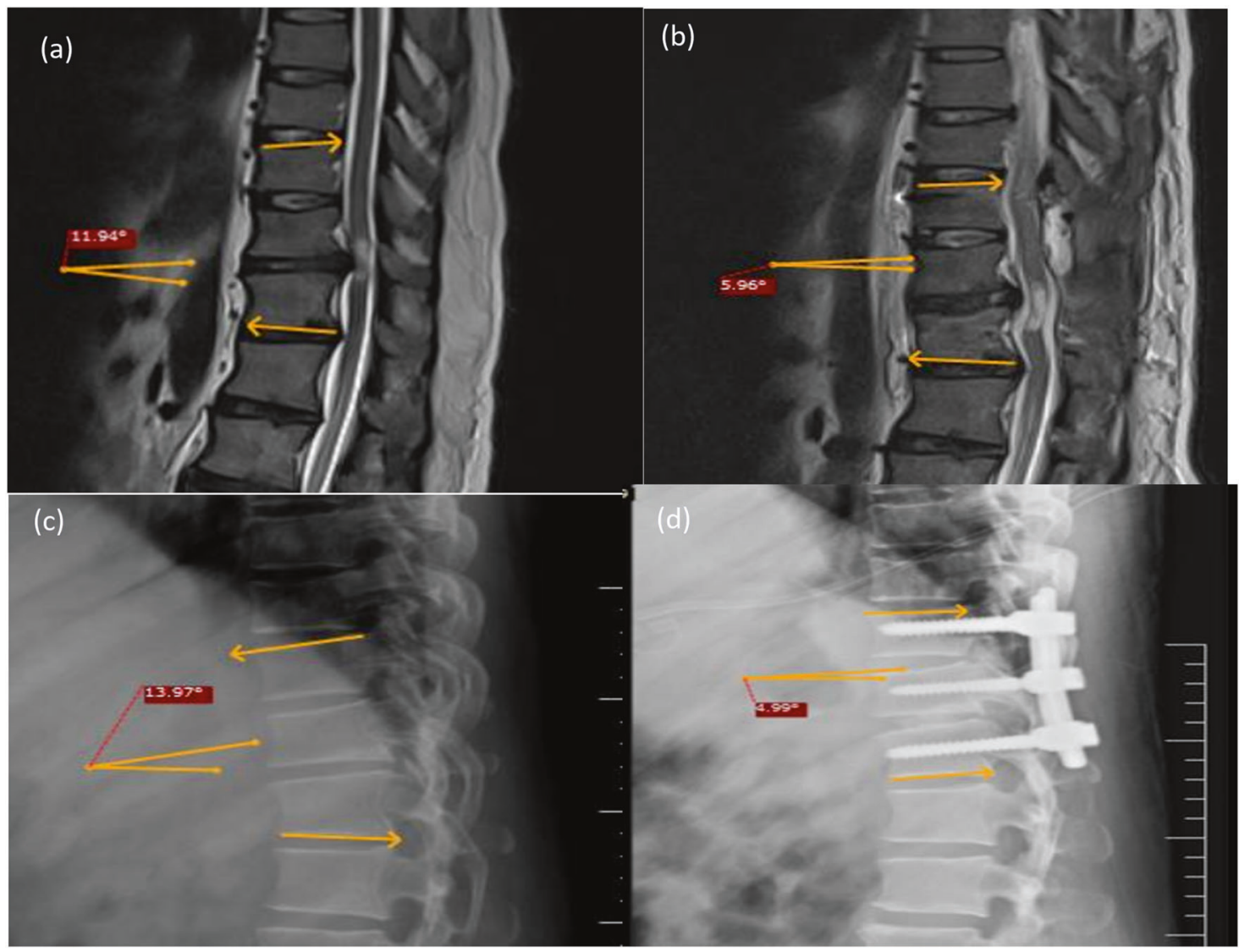

Fig. 6 Preoperative (a) and postoperative (b) T2 weighted MRI images of a 71-year-old male (case 4) with dilated cardiomyopathy who presented to us with severe progressive thoracic myelopathy showing adequate fall back of the cord. CSF column could be appreciated in front of the involved spinal cord. The segmental kyphosis at the level

Acknowledgements The authors would like to thank the concerned patients for allowing the details to be shared.

\section{Compliance with ethical standards}

Conflict of interest The authors declare that they have no conflict of interest.

Publisher's note: Springer Nature remains neutral with regard to jurisdictional claims in published maps and institutional affiliations.

\section{References}

1. Yonenobu K, Ebara S, Fujiwara K, Yamashita K, Ono K, Yamamoto T. Thoracic myelopathy secondary to ossification of the spinal ligament. J Neurosurg. 1987;66:511-8. https://www. ncbi.nlm.nih.gov/pubmed/3104552.

2. Sato T, Kokubun S, Tanaka Y, Ishii Y. Thoracic myelopathy in the Japanese: epidemiological and clinical observations on the cases in Miyagi Prefecture. Tohoku J Exp Med. 1998;184:1-11. https://www.ncbi.nlm.nih.gov/m/pubmed/9607393/.

3. Maiman DJ, Pintar FA. Anatomy and clinical biomechanics of the thoracic spine. Clin Neurosurg. 1992;38:296-324. https://www. ncbi.nlm.nih.gov/pubmed/1537190. of instrumentation (T10 to T12) decreased from $11.9^{0}$ preoperatively to $6^{0}$ postoperatively. Preoperative (c) and postoperative (d) X-ray images of the patient (case 4) showing that the segmental kyphosis between T10 to T12 decreased from $14^{0}$ preoperatively to $5^{0}$ postoperatively. He had a good improvement in symptoms postoperatively

4. Chen Z, Sun C. Clinical guideline for treatment of symptomatic thoracic spinal stenosis: guideline for treatment symptomatic TSS. Orthop Surg 2015;7:208-12.

5. Imagama S, Ando K, Takeuchi K, Kato S, Murakami H, Aizawa T. Perioperative complications after surgery for thoracic ossification of posterior longitudinal ligament: a nationwide multicenter prospective study. Spine (Philos Pa 1976). 2018;43:E1389-E1397. https://journals.lww.com/spinejournal/Abstract/2018/12010/ Perioperative_Complications_After_Surgery_for.6.aspx.

6. Tsuzuki N, Hirabayashi S, Abe R, Saiki K. Staged spinal cord decompression through posterior approach for thoracic myelopathy caused by ossification of posterior longitudinal ligament. Spine (Phila Pa 1976). 2001;26: 1623-30. https://www.ncbi.nlm. nih.gov/pubmed/11464158.

7. Fujimura $\mathrm{Y}$, Nishi $\mathrm{Y}$, Nakamura M, Watanabe M, Matsumoto M. Myelopathy secondary to ossification of the posterior longitudinal ligament of the thoracic spine treated by anterior decompression and bony fusion. Spinal Cord. 1997;35:777-84. https://www.na ture.com/articles/3100487.

8. Takahata M, Ito M, Abumi K, Kotani Y, Sudo H, Minami A. Clinical results and complications of circumferential spinal cord decompression through a single posterior approach for thoracic myelopathy caused by ossification of posterior longitudinal ligament. Spine (Philos Pa 1976). 2008;33:1199-208. https://www. ncbi.nlm.nih.gov/pubmed/18469693. 
9. Tomita K, Kawahara N, Baba H, Kikuchi Y, Nishimura H. Circumspinal decompression for thoracic myelopathy due to combined ossification of the posterior longitudinal ligament and ligamentum flavum. Spine (Philos Pa 1976). 1990;15:1114-20. https://www.ncbi.nlm.nih.gov/pubmed/2125147.

10. Tsuzuki N, Wadano Y, Kikuchi S-i. Extensive cervicothoracic laminoplastic decompression of the spinal cord: a new method of posterior decompression for thoracic myelopathy caused by ossification of the posterior longitudinal ligament. In: Yonenobu K, Sakou T, Ono K, editors OPLL. Tokyo: Springer; 1997. https:// link.springer.com/chapter/10.1007\%2F978-4-431-67046-9_22.

11. Yamazaki M, Okawa A, Koda M, Goto S, Minami S, Moriya H. Transient paraparesis after laminectomy for thoracic myelopathy due to ossification of the posterior longitudinal ligament: a case report. Spine (Philos Pa 1976). 2005;30:E343-6. https://www. ncbi.nlm.nih.gov/pubmed/15959359.

12. Yamazaki M, Mochizuki M, Ikeda Y, Sodeyama T, Okawa A, Koda M. et al. Clinical results of surgery for thoracic myelopathy caused by ossification of the posterior longitudinal ligament: operative indication of posterior decompression with instrumented fusion. Spine (Philos Pa 1976). 2006;31:1452-60. https://www. ncbi.nlm.nih.gov/pubmed/16741454.

13. Matsuyama $\mathrm{Y}$ et al. Surgical treatment for ossification of the posterior longitudinal ligament of the thoracic spine: outcomes of one-stage posterior decompression with corrective fusion surgery. In: Yonenobu K, Nakamura K, Toyama Y, editors OPLL. Tokyo: Springer; 2006. 10.1007/978-4-431-32563-5_36. https://link. springer.com/chapter/10.1007/978-4-431-32563-5_36.

14. Matsuyama Y, Sakai Y, Katayama Y, Imagama S, Ito Z, Wakao N, et al. Indirect posterior decompression with corrective fusion for ossification of the posterior longitudinal ligament of the thoracic spine: is it possible to predict the surgical results? Eur Spine J. 2009;18:943-8. https://doi.org/10.1007/s00586-009-0956-2.

15. Kawahara N, Tomita K, Murakami H, Hato T, Demura S, Sekino Y. et al. Circumspinal decompression with dekyphosis stabilization for thoracic myelopathy due to ossification of the posterior longitudinal ligament. Spine. 2008;33:39-46. https://doi.org/10. 1097/BRS.0b013e31815e3911. 